Open Access

\title{
Targeted next-generation sequencing reveals high frequency of mutations in epigenetic regulators across treatment-naïve patient melanomas
}

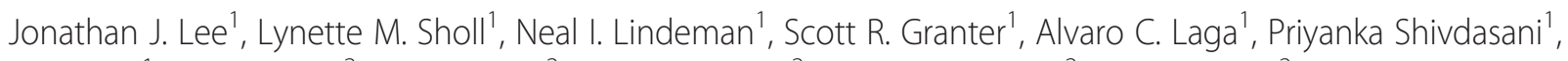
Gary Chin ${ }^{1}$, Jason J. Luke ${ }^{2}$, Patrick A. Ott ${ }^{2}$, F. Stephen Hodi ${ }^{2}$, Martin C. Mihm Jr. ${ }^{2}$, Jennifer Y. Lin², Andrew E. Werchniak²,

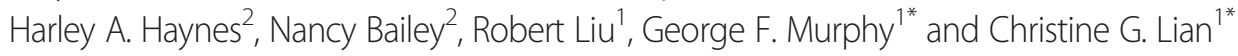

\begin{abstract}
Background: Recent developments in genomic sequencing have advanced our understanding of the mutations underlying human malignancy. Melanoma is a prototype of an aggressive, genetically heterogeneous cancer notorious for its biologic plasticity and predilection towards developing resistance to targeted therapies. Evidence is rapidly accumulating that dysregulated epigenetic mechanisms (DNA methylation/demethylation, histone modification, non-coding RNAs) may play a central role in the pathogenesis of melanoma. Therefore, we sought to characterize the frequency and nature of mutations in epigenetic regulators in clinical, treatment-naïve, patient melanoma specimens obtained from one academic institution.

Results: Targeted next-generation sequencing for 275 known and investigative cancer genes (of which 41 genes, or $14.9 \%$, encoded an epigenetic regulator) of 38 treatment-naïve patient melanoma samples revealed that $22.3 \%$ (165 of 740) of all non-silent mutations affected an epigenetic regulator. The most frequently mutated genes were BRAF, MECOM, NRAS, TP53, MLL2, and CDKN2A. Of the 40 most commonly mutated genes, 12 (30.0\%) encoded epigenetic regulators, including genes encoding enzymes involved in histone modification (MECOM, MLL2, SETD2), chromatin remodeling (ARID1B, ARID2), and DNA methylation and demethylation (TET2, IDH1). Among the 38 patient melanoma samples, 35 (92.1\%) harbored at least one mutation in an epigenetic regulator. The genes with the highest number of total UVB-signature mutations encoded epigenetic regulators, including MLL2 (100\%, 16 of 16) and MECOM (82.6\%, 19 of 23). Moreover, on average, epigenetic genes harbored a significantly greater number of UVB-signature mutations per gene than non-epigenetic genes (3.7 versus 2.4 , respectively; $p=0.01$ ). Bioinformatics analysis of The Cancer Genome Atlas (TCGA) melanoma mutation dataset also revealed a frequency of mutations in the 41 epigenetic genes comparable to that found within our cohort of patient melanoma samples.

(Continued on next page)
\end{abstract}

\footnotetext{
* Correspondence: gmurphy@rics.bwh.harvard.edu; cglian@partners.org ${ }^{1}$ Department of Pathology, Brigham and Women's Hospital, Harvard Medical School, 221 Longwood Avenue, EBRC Suite 401, Boston, MA 02115, USA Full list of author information is available at the end of the article
} 
(Continued from previous page)

Conclusions: Our study identified a high prevalence of somatic mutations in genes encoding epigenetic regulators, including those involved in DNA demethylation, histone modification, chromatin remodeling, and microRNA processing. Moreover, UVB-signature mutations were found more commonly among epigenetic genes than in non-epigenetic genes. Taken together, these findings further implicate epigenetic mechanisms, particularly those involving the chromatin-remodeling enzyme MECOM/EVI1 and histone-modifying enzyme MLL2, in the pathobiology of melanoma.

Keywords: Melanoma, Next-generation sequencing (NGS), Epigenetics, MECOM (MDS1 and EV1 complex locus), MLL2, Ten-eleven translocation (TET), Isocitrate dehydrogenase 2 (IDH2), 5-hydroxymethylcytosine, DNA demethylation

\section{Background}

Despite advancements in our understanding of the molecular mechanisms underlying melanogenesis, disease progression, and therapeutic response, melanoma remains one of the deadliest forms of human malignancy [1]. Identification of frequent mutations in the gene encoding the serine/ threonine-protein kinase B-Raf $(B R A F)$ in melanoma [2] led to the development of targeted inhibitors with improved survival demonstrated in phase III clinical trials demonstrating improved overall survival [3]. However, soon after the discovery of frequent BRAF V600E mutations in melanoma, the same mutation was found at higher frequencies in benign and dysplastic melanocytic nevi [4]. Since then, $B R A F$ mutations, alone, have become understood to be "insufficient" to induce tumor progression beyond the benign melanocytic nevus stage [5].

Despite initial clinical response after targeted, single agent therapy, the subsequent development of resistance in patients being treated for metastatic melanoma is, essentially, universal [6]. This is largely attributed to the development and progression of chemoresistant subpopulations, enabled, in part, by unique biological characteristics inherent to malignant melanoma [7]. Complicating matters is that approximately one in every five patients harboring $B R A F$ V600 mutant melanoma will have disease that is intrinsically resistant to $B R A F$ inhibition and will be found to have progressed on therapy at first follow-up assessment [6]. While the combined use of targeted therapies (BRAF and/or MEK inhibition) [7-9] as well as immunotherapies (monoclonal antibodies directed at CTLA-4 and PD-1/PDL1) have shown significantly improved outcomes for melanoma patients with advanced disease [10, 11], evidence implicating dysregulated epigenetic mechanisms in the pathogenesis of melanoma and other malignancies is also accumulating at a rapid pace $[12,13]$. This growing body of literature has significant translational potential to elucidate novel pathogenic mechanisms in melanoma and deserves thorough investigation, as therapeutically targeting epigenetic mechanisms $[14,15]$ in combination with other targeted or immunotherapeutic modalities may be necessary to achieve sustainable clinical remission [16-18].
Recent advancements in next-generation sequencing (NGS) technologies have facilitated whole-genome, wholeexome, and whole-transcriptome analyses that could enable personalized diagnostic and therapeutic strategies. These technologies have demonstrated substantial power and sensitivity in identifying novel mutated genes in melanoma and have even been used to gain insight into mechanisms of primary (intrinsic) [17] and secondary (acquired) chemoresistance in select melanoma patients $[7,19]$. Moreover, developments in computational processing and statistical analytics have also enabled the dissection of "driver" mutations (those that confer a fitness advantage to a particular tumor cell) from "passenger" mutations (those that do not) [20]. Indeed, such efforts to enhance molecular precision while characterizing the genetic and epigenetic landscape within an individual patient's melanoma may be necessary to help guide combinatorial therapies [21].

Herein, we describe the targeted NGS platform devised at our institution to sequence 275 known and investigative cancer genes ("Oncopanel”, Brigham and Women's Hospital and Dana-Farber Cancer Institute) in patients being evaluated for the management of melanoma and report the frequency and nature of gene mutations identified. A list of the genes tested for by the Oncopanel platform is provided in Additional file 1. A substantial fraction (14.9\%, 41 of 275) of these genes encode either well-established or recently-described epigenetic regulators, including those involved in DNA methylation and demethylation, histone modification, chromatin remodeling, and non-coding RNAs. In light of rapidly accumulating evidence for the involvement of dysregulated epigenetic mechanisms in melanoma pathogenesis [12, 13], we sought, specifically, to characterize the prevalence and nature of mutations in this select panel of epigenetic regulators within our cohort of patient melanoma samples.

\section{Results}

Patient demographic, clinical, and histopathologic information

A total of 38 patient melanoma samples $(n=38)$, each obtained from 38 unique patients, were available for analysis. 
Of these, 13 were primary cutaneous melanomas $\left(n_{\mathrm{P}}=13\right)$ and the remaining 25 were obtained from metastatic sites $\left(n_{\mathrm{M}}=25\right)$. Patients ranged in age from 21 to 83 years (mean, 60.9; median, 62; SD, 13.06). Primary cutaneous melanoma samples ranged in Breslow depth and mitotic rate from 0.67 to $7.50 \mathrm{~mm}$ (mean, $3.66 \mathrm{~mm}$; median, 3.1; $\mathrm{SD}, 1.94$ ) and from 1 to 17 mitoses $/ \mathrm{mm}^{2}$ (mean, 5.3; median, 4; SD, 4.5). Six of the primary melanomas (46.2 \%, 6 of 13) had histologic evidence of ulceration. Of the patients whose primary melanomas had been sequenced, five (38.5\%) had also gone on to develop metastatic disease. The vast majority of metastatic melanoma tissues samples were obtained from lymph nodes (44 \%, 11 of 25$)$, while the remaining metastases were obtained from the thorax (i.e., lung, chest wall, $16 \%$, 4 of 25), abdomen (i.e., mesentery, adrenal gland, 4 of $25,16 \%$ ), central nervous system (i.e., brain, brainstem, $12 \%, 3$ of 25 ), or subcutaneous tissue (i.e., in transit metastasis) (12\%, 3 of 25 ).

\section{General mutation distribution and characteristics}

Collectively across all 38 patient samples, a total of 740 non-silent mutations were identified in 204 of the 275 (74.2\%) genes originally tested for. An average of approximately 20 mutations (median, 15.5; range, 3 to 132; standard deviation, 21.5) was identified per patient melanoma sample. A graph summarizing the distribution of mutation types is shown in Fig. 1. The vast majority of mutations were missense mutations $(84.7 \%, 627$ of 740$)$, followed by nonsense mutations $(8.9 \%, 66$ of 740$)$, insertions or deletions resulting in frameshift $(2.0 \%, 14$ of 740$)$, and splice site mutations $(2.0 \%, 15$ of 740$)$. The largest percentage of nonsense mutations occurred in well-known tumor suppressor genes NF1 (12.1\%, 8 of 66), CDKN2A (10.6\%, 7 of 66), and TP53 (9.1\%, 6 of 66).

The top 40 most frequently mutated genes, as determined by the number and frequency of total mutations, are graphically represented in Fig. 2. BRAF $(42.1 \%$ of patient samples, 16 of 38), MECOM (36.8\%, 14 of 38), NRAS (36.8 \%, 14 of 38), TP53 (31.6 \%, 12 of 38), MLL2 (29.0\%, 11 of 38$)$, as well as CDKN2A $(29.0 \%, 11$ of 38$)$ were among the most commonly mutated genes among the patient melanoma samples. Of these genes, MECOM, $B R A F$, and $M L L 2$ harbored the greatest number of total mutations $(23,19,16$, respectively).

\section{High frequency of mutations identified in key epigenetic regulators}

Of interest, $22.3 \%$ (165 of 740) of all mutations occurred in genes encoding epigenetic regulators. Mutations in genes encoding histone-modifying proteins were the most common (64.2\%, or 106 of 165 epigenetic gene mutations, which accounted for $14.3 \%$, or 106 of all 740 identified mutations), including $M E C O M$ and $M L L 2$ listed above, followed by chromatin remodeling proteins $(24.2 \%, 40$ of 165), DNA methylation/demethylation enzymes (9.1\%, 15 of 165), and enzymes involved in miRNA processing $(2.4 \%, 4$ of 165$)$. A summary of the frequency of mutated epigenetic genes categorized by their functional epigenetic

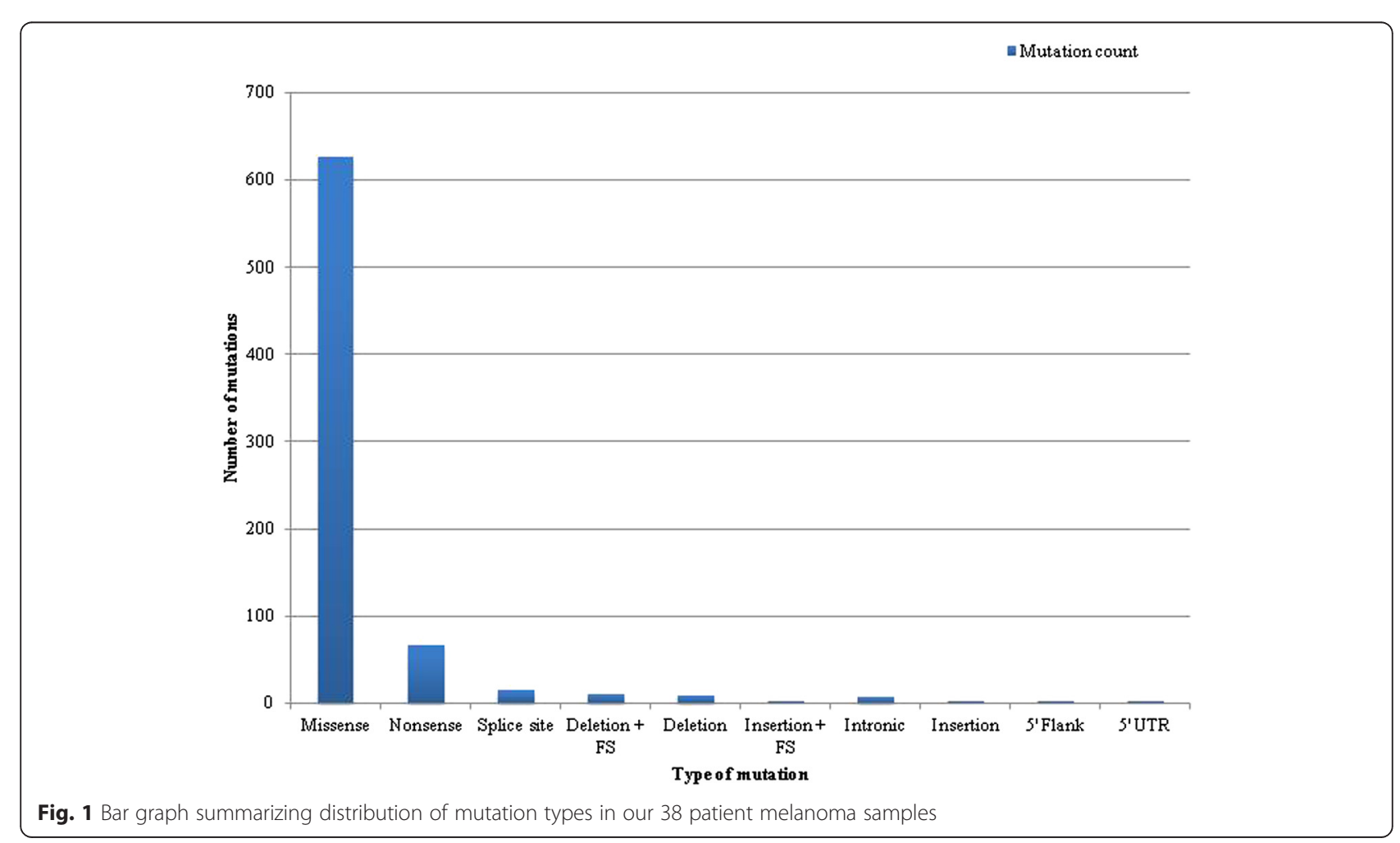




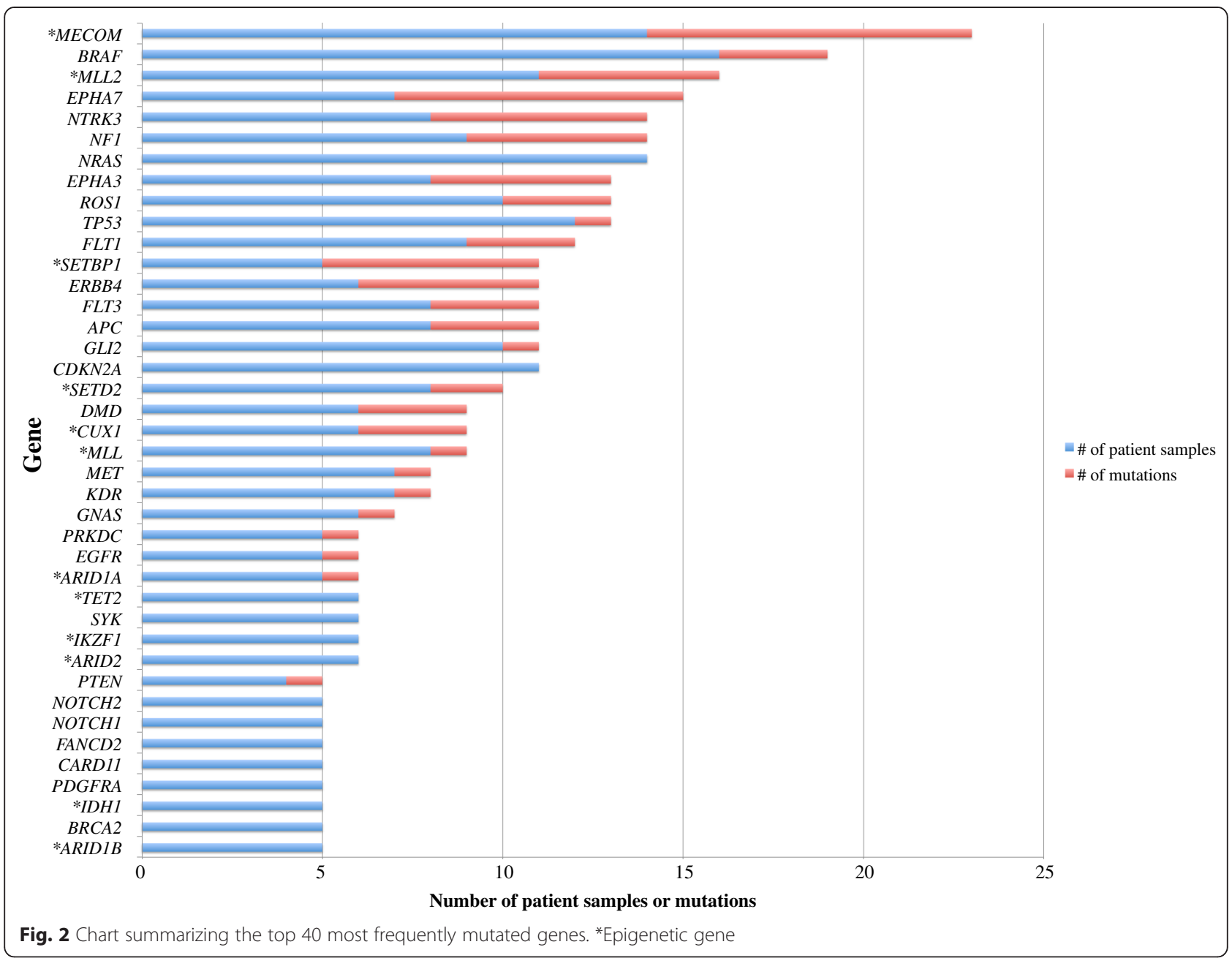

classification is illustrated in Fig. 3. At least one mutation in an epigenetic gene was found in $92.1 \%$ (35 of 38) of patients, and 25 of these patients ( $65.7 \%$ of all samples) had more than one epigenetic regulatory gene mutated.

Of all the mutated genes identified, $17.2 \%$ (35 of 204) encoded epigenetic regulators, whereas $14.9 \%$ (41 of 275) of the genes tested by our Oncopanel were epigenetic in nature. Moreover, within the top 40 most frequently mutated genes, $30.0 \%$ (12 of 40) of the genes encoded epigenetic regulators (Fig. 2). A two-sample $z$ test comparing the proportion of epigenetic regulators within the top 40 (30\%, 12 of 40) compared to that within the original panel of tested genes $(14.9 \%, 41$ of 275$)$ revealed a statistically significant difference $(p=0.017, z$ score $=2.4)$ between these two groups. Ten of $12(81.2 \%)$ of the epigenetic genes within the top 40 were found to encode either histone-modifying proteins (e.g., MECOM, MLL2, SETD2) or subunits of chromatin-remodeling complexes (e.g., ARID1B, ARID2). Two of 12 (16.7\%) of these epigenetic genes within the top 40 encode enzymes involved in active DNA demethylation (TET2, IDH1). Furthermore, $30.8 \%$
(4 of 13) of all genes containing an insertion or deletion resulting in frameshift encoded epigenetic regulators, including histone-modifying enzymes (SETD2, CREBBP, $M L L)$ and DNA methyltransferase 3A DNMT3A.

\section{Analysis of epigenetic mutations and key clinicopathologic parameters}

A summary graphic illustrating the spectrum of epigenetic genes mutated within each patient melanoma sample as well as the clinical and histopathologic data corresponding to each patient's case is presented in Fig. 4. Our analysis did not identify statistically significant relationships between mutations in specific epigenetic genes and key histologic parameters of primary cutaneous melanoma, such as the Breslow depth, mitotic rate, or the presence/ absence of ulceration; nor did it reveal any specific epigenetic mutational signature of either primary or metastatic melanomas within the limited number of samples in our study. Nonetheless, ingenuity pathway analysis revealed a complex mechanistic interplay between several of our most frequently mutated epigenetic regulators and the 


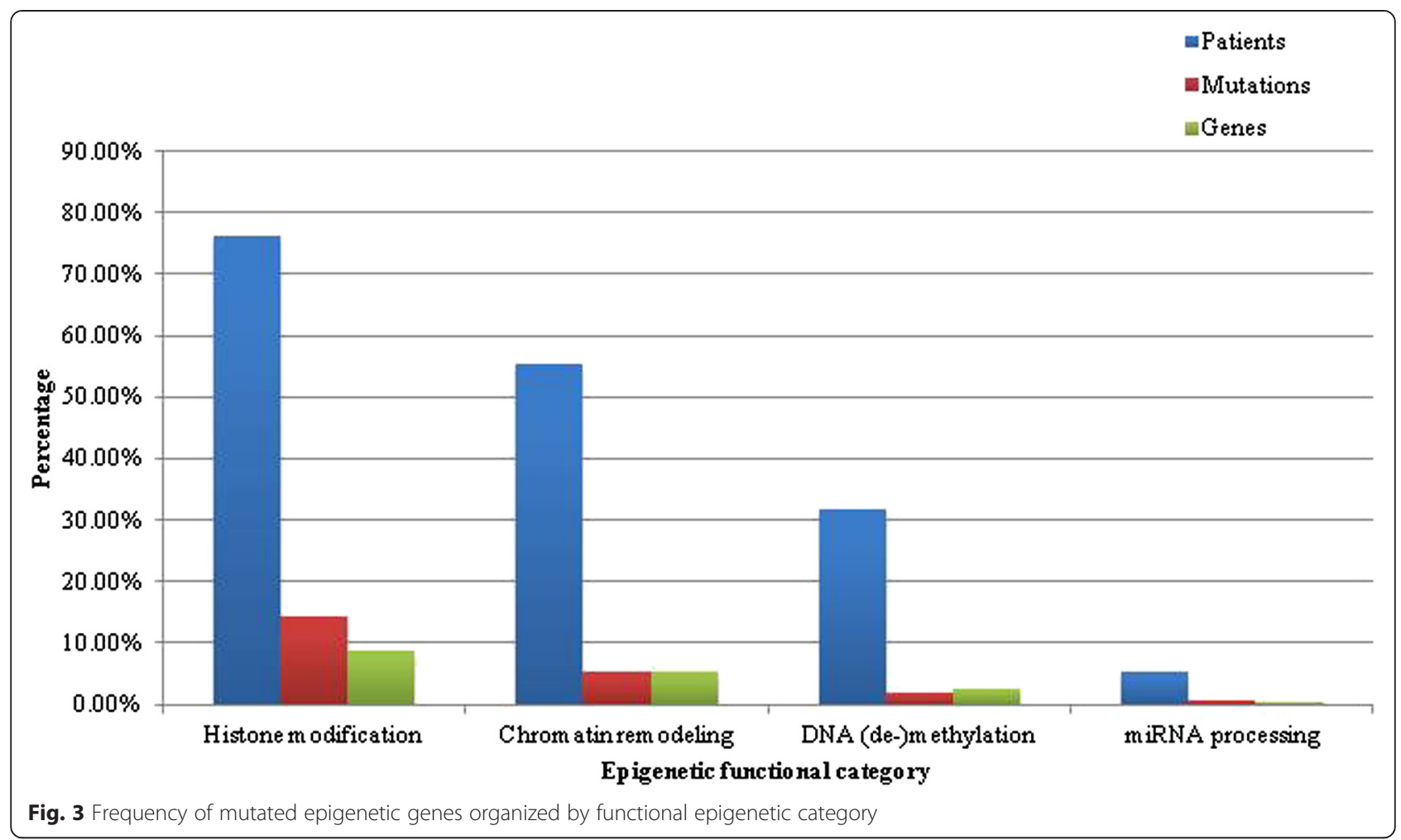

prevalence of mutations in each epigenetic regulator is also graphically represented (Fig. 5).

\section{High frequency of UVB-pattern mutations particularly found among key epigenetic regulators}

Of all the mutations identified, $73.1 \%$ (541 of 740) bore the signature of UVB damage. A single $\mathrm{C}>\mathrm{T}$ nucleotide substitution was the most common (70.3\%, 520 of 740), followed by $\mathrm{CC}>\mathrm{TT}$ tandem dinucleotide mutation (2.2\%, 12 of 541 ), and $\mathrm{C}>\mathrm{T}$ missense mutations within a dinucleotide substitution (1.7\%, 9 of 541). In contrast, only $4.9 \%$ (36 of 740 ) of all mutations were $\mathrm{G}>\mathrm{T}$ single nucleotide variant transitions, the signature of UVAinduced DNA damage. The genes with the greatest number of total UVB-signature mutations encoded epigenetic regulators, including $M L L 2$ (100\%, 16 of 16) and MECOM (82.6 \%, 19 of 23). The spectrum of UVsignature mutations in non-epigenetic and epigenetic genes is provided in Figs. 6 and 7, respectively.

Collectively, non-epigenetic genes harbored a mean of 2.4 UVB-signature mutations per gene (median, 2; mode, 1 ; standard deviation, 2.5) whereas epigenetic genes harbored an average of 3.7 (median, 3; mode, 3; standard deviation, 3.9) (Fig. 8). An unpaired, two-sample $t$ test comparing the average number of UVB-signature mutations per gene in non-epigenetic versus epigenetic genes revealed a statistically significant difference $(p=0.014)$ between the two groups. Of note, $81.8 \%$ (9 of 11) of
CDKN2A mutations and $84.6 \%$ (11 of 13) of TP53 mutations bore the UVB-signature. Interestingly, none of the NRAS mutations ( 0 of 14) and only $47.4 \%$ (9 of 19) of $B R A F$ mutations resembled the UVB-signature mutation.

\section{Bioinformatics analysis of existing melanoma mutation datasets}

Targeted analysis of melanoma mutation data, publicly available online through The Cancer Genome Atlas database (TCGA, http://www.cbioportal.org) was also performed on 278 melanoma samples provided by the TCGA $\left(n_{\mathrm{T}}=278\right)$ and 121 samples made available through the Broad Institute (Cambridge, MA) $\left(n_{\mathrm{B}}=121\right)$ $[22,23]$. We found that the frequency of mutations in genes among our top 40 most frequently mutated was, overall, comparable to that found in $97.1 \%$ (270 of 278) of TCGA melanoma samples (Fig. 9) and $95 \%$ (115 of 121) of samples provided by the Broad Institute (Fig. 10), although eight of our top 40 (20\%) genes were not available through this database. Notably, however, the prevalence of BRAF mutations was higher in the TCGA and Broad Institute cohorts (51 and $63 \%$, respectively) than in our dataset (42\%). In addition, MECOM mutations were found less frequently in the TCGA and Broad Institute cohorts (20 and $16 \%$, respectively).

Furthermore, a focused analysis of mutations in the 41 epigenetic genes tested by our Oncopanel in the TCGA 


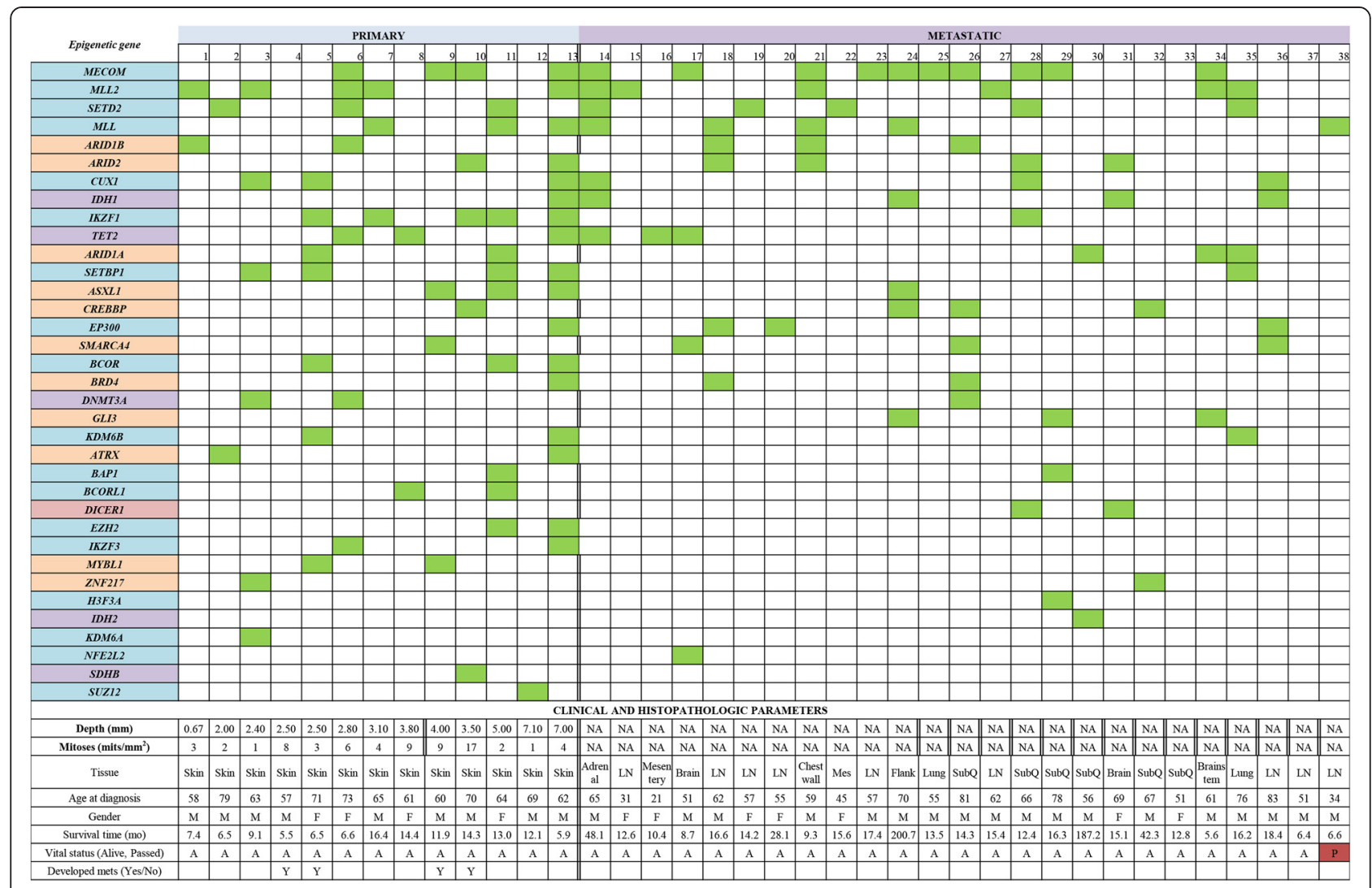

Fig. 4 Presence of mutations in epigenetic regulators, organized by patient melanoma sample and key primary cutaneous melanoma histopathologic parameters

and Broad cohorts revealed a comparable frequency of mutations (Figs. 11 and 12). In keeping with our data, MECOM, MLL2 (KMT2D), MLL (KMT2A), ARID2, and CUX1 were among the most frequently mutated epigenetic genes in the TCGA and Broad Institute melanoma cohorts. In addition, the vast majority of samples in both the TCGA (83.1\%, 231 of 278) and Broad Institute $(69.4 \%, 84$ of 121) cohorts harbored at least one mutation in one of the tested epigenetic genes.

\section{Discussion}

The incidence of melanoma, unlike many other potentially preventable cancers, is steadily increasing worldwide, with an estimated 76,100 new cases diagnosed in the USA in 2014 alone [1]. While accounting for less than $2 \%$ of all skin cancers, melanoma accounts for the vast majority of skin cancer deaths [1]. Major risk factors for melanoma include those that are genetic and environmental in nature, such as having a personal or family history of melanoma, five or more "atypical" nevi, having numerous (>50) melanocytic nevi, fair-colored skin, as well as either a history of blistering sun burns during childhood/adolescence and/or a history of indoor tanning bed use [24]. Epidemiological evidence increasingly implicates UV radiation in melanoma pathogenesis, as was recently detailed in a meta-analysis that demonstrated an increased risk of melanoma in airline pilots and cabin crew (thought to be related to elevated levels of cosmic and UV radiation exposure) [25]. Approximately $10 \%$ of melanomas occur in a familial setting, and germline mutations to a number of genes, including CDKN2A (9p21), CDK4 (12q14), BAP1 (3p21), TERT promoter (5p15) [26], and most recently POT1 (7q31) [27], have been demonstrated to predispose individuals to developing cutaneous melanoma in addition to other melanoma subtypes (e.g., $B A P 1$ and metastatic uveal melanoma) as well as numerous atypical melanocytic nevi (CDKN2A, CDK4) [28]. Collectively, these observations reinforce that the pathogenesis of melanoma is complex, involving both genetic and non-heritable (e.g., environmental) factors.

The most frequently mutated genes within our cohort resemble those reported in previous studies [20,29] as well as through data available through the Sanger Institute Catalogue Of Somatic Mutations in Cancer (COSMIC) database (http://www.sanger.ac.uk/) and the NIH/NCI TCGA melanoma mutation database (http://www.cbio portal.org) [22, 23, 30]. The well-known oncogenes NRAS and $B R A F$ as well as the tumor suppressor genes $C D K N 2 A$ 


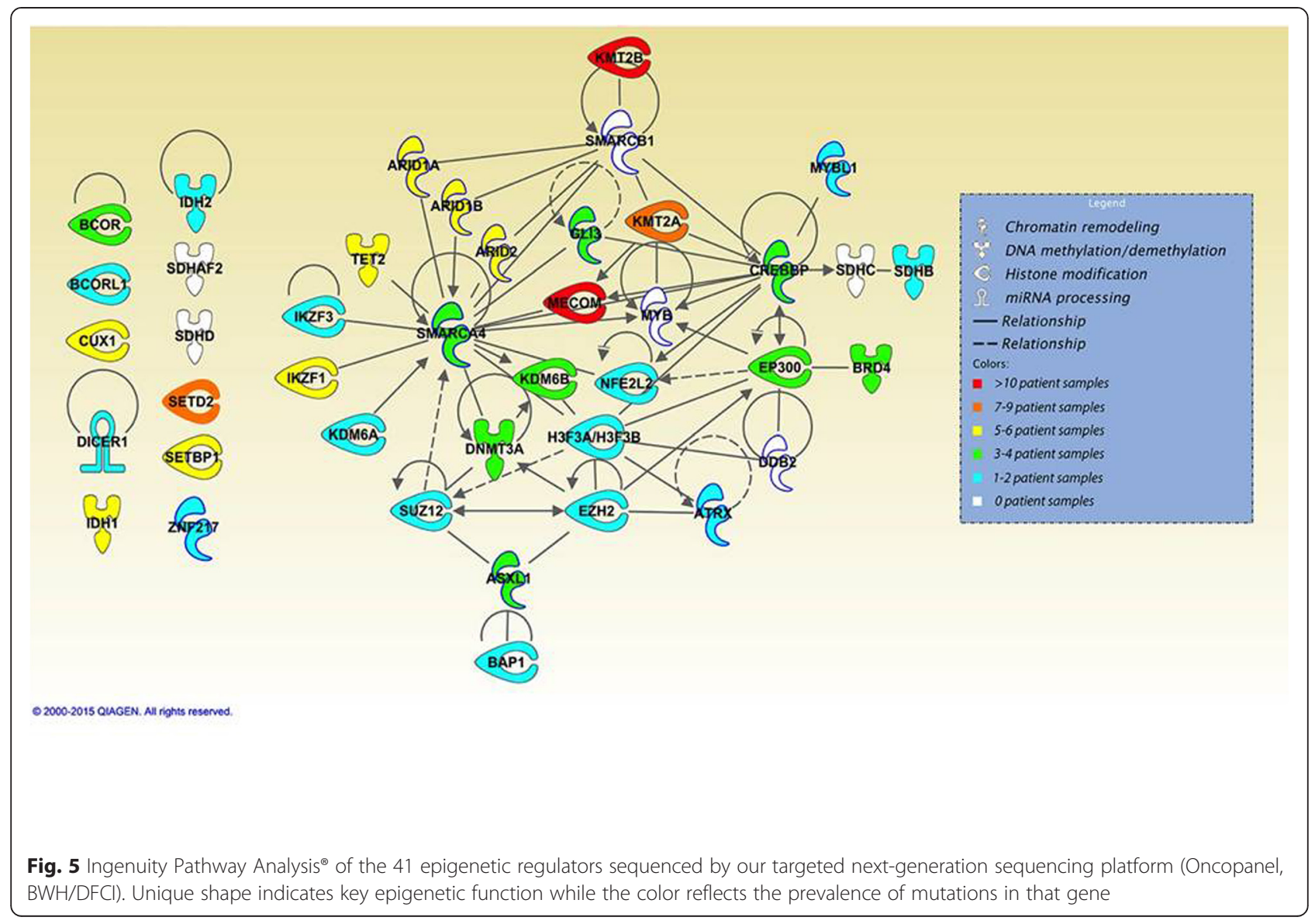

and TP53 (17p13) were among the most frequently mutated genes within our cohort, in keeping with prior studies and datasets [20,31]. The latter two genes were also among the genes within our cohort containing the highest number and percentage of nonsense mutations, consistent with loss of their tumor suppressive function. While mutations to the TP53 gene have previously been considered a rare event in melanomagenesis [32], our findings corroborate data from others [20] demonstrating that they may be more common than previously believed. While these large, publically available datasets have the advantage of pooling larger number of patient melanoma samples, at present, they do not allow for the specific characterization of UV-signature mutations, nor do they allow for the direct comparison of such patterns or others between epigenetic and nonepigenetic genes, as our study has done. In addition, these databases do not allow for potential relationships between specific mutations and key melanoma histologic parameters to be explored.

Our dataset and novel analytic approach, with an emphasis on epigenetic mutations, reveal several interesting observations. Firstly, we identified a high frequency of mutations in genes encoding epigenetic regulators in both primary and metastatic cutaneous melanoma patient samples with a statistically significant predilection for mutations bearing the signature of UVB damage. Genes encoding histone-modifying proteins (e.g., $M E C O M, M L L 2$, SETD2, etc.), subunits of chromatin-remodeling complexes (e.g., ARID1B, ARID2), as well as units of the active DNA demethylation pathway $(T E T 2, I D H 1)$ were the most frequently mutated among this list. We took particular interest in several novel standouts among our list of most commonly mutated epigenetic genes. MECOM (3q26), for instance, was among the most frequently mutated genes (3.1\%, 23 of 740 mutations; $36.8 \%$, 14 of 38 patient samples) within our cohort of patient melanoma samples and was higher than estimated by existing datasets (Figs. 9 and 10) [30].

MECOM (MDS1 and EV1 complex locus) encodes ecotropic viral integration site 1 (EVI1), an oncogenic zinc finger transcription factor known to be overexpressed in acute and chronic myeloid leukemia and to correlate with poor patient survival [33, 34]. Interactome analysis has revealed that the EVI1 oncoprotein exerts dynamic nuclear functions and is involved in a number of vital processes, including, but not limited to, transcription regulation, DNA repair, recombination, and mitosis [35]. In addition, EVI1 has been shown to interact with 


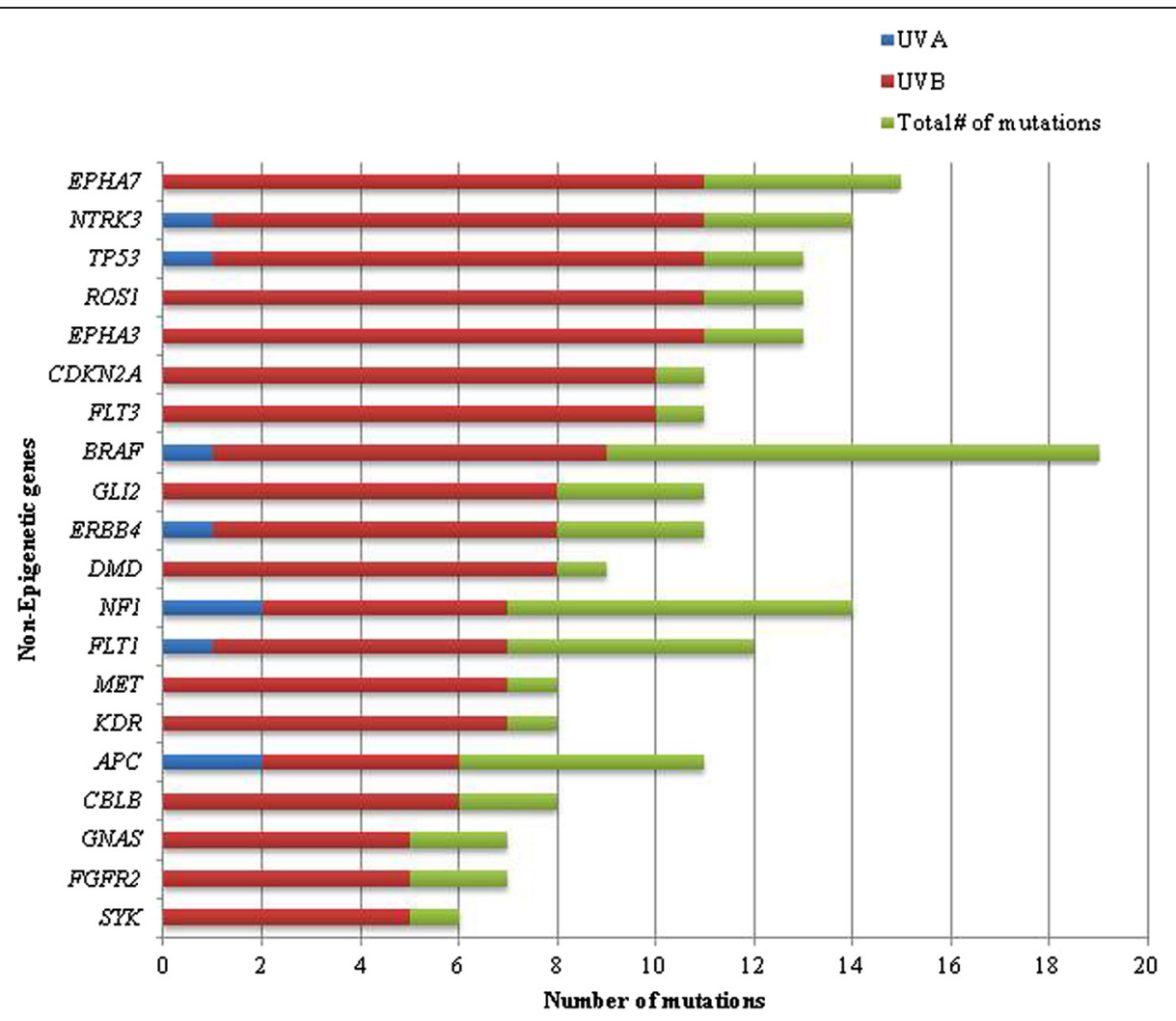

Fig. 6 Spectrum of UV-signature mutations among non-epigenetic genes. Note the low frequency of UVB-signature mutations in BRAF

multiple components of the epigenetic machinery, including DNA methyltransferases, histone modifying enzymes, and chromatin-remodeling complexes, including the SWItch/Sucrose NonFermentable (SWI/SNF) nucleosome remodeler [36]. Moreover, gene expression analyses demonstrated a stem cell phenotype in EVI1-overexpressing acute myelocytic leukemia cells, suggesting that this oncoprotein could augment cancer stem cell self-renewal capacity and facilitate disease progression and the development of therapeutic resistance [36]. Similar lines of evidence also suggest that EVI1 may be involved in facilitating chemoresistance in human myeloid leukemias by inducing the CDKN1A/p21/WAF complex [37]. Taken together, we hypothesize that MECOM/EVI1 regulates the epigenetic machinery enabling stem cell-like properties in specific melanoma subpopulations. Additional studies are indicated to explore and evaluate these possibilities.

MLL2 (or KMT2D, 12q13) was the second most frequently mutated epigenetic gene within our cohort. MLL2 is a member of the myeloid/lymphoid or mixed-lineage leukemia $(M L L)$ family genes and encodes a specific histone 3 lysine 4 (H3K4) methyltransferase, which provides an evolutionarily conserved epigenetic mark for active gene transcription [38]. Remarkably, all 16 of 16 mutations in $M L L 2$ bore the signature of UVB-induced DNA damage. $M L L 2$ was recently identified to extensively regulate the expression of a number of critical cell signaling pathways, including the p53 pathway and cAMP-mediated signaling, as well as the expression of the retinoic acidresponsive gene ASB2 [39]. Moreover, and of particular interest to the biology of melanocytes and melanoma, $M L L 2$ was recently found to associate with the promoters and thereby regulate the expression of S100 alpha (S100A) genes (1q21), which are known to control cell cycle progression and differentiation within the melanocyte [39]. MLL2 has been frequently implicated in the pathogenesis of a number of human cancers [40-42], and our findings corroborate with recent data [31] suggesting the same may be true for malignant melanoma.

The $I D H 1$ gene was also among the most commonly mutated epigenetic genes within our collection and was more frequently mutated than reported in prior studies [20]. IDH1 (2q33) encodes a soluble, cytoplasmic isocitrate dehydrogenase 1 (IDH1) enzyme, an enzyme that converts isocitrate to $\alpha$-ketoglutarate (also known as 2-oxoglutarate) [43]. Recent evidence suggests significant epigenetic consequences resulting from mutations in this particular enzyme. Loss of IDH1 function results in reduced production of $\alpha$-ketoglutarate, which is a necessary co-substrate for two critical families of epigenetic regulators, including histone demethylase enzymes and the ten-eleven translocation (TET) family of 5-methylcytosine hydroxylases, 


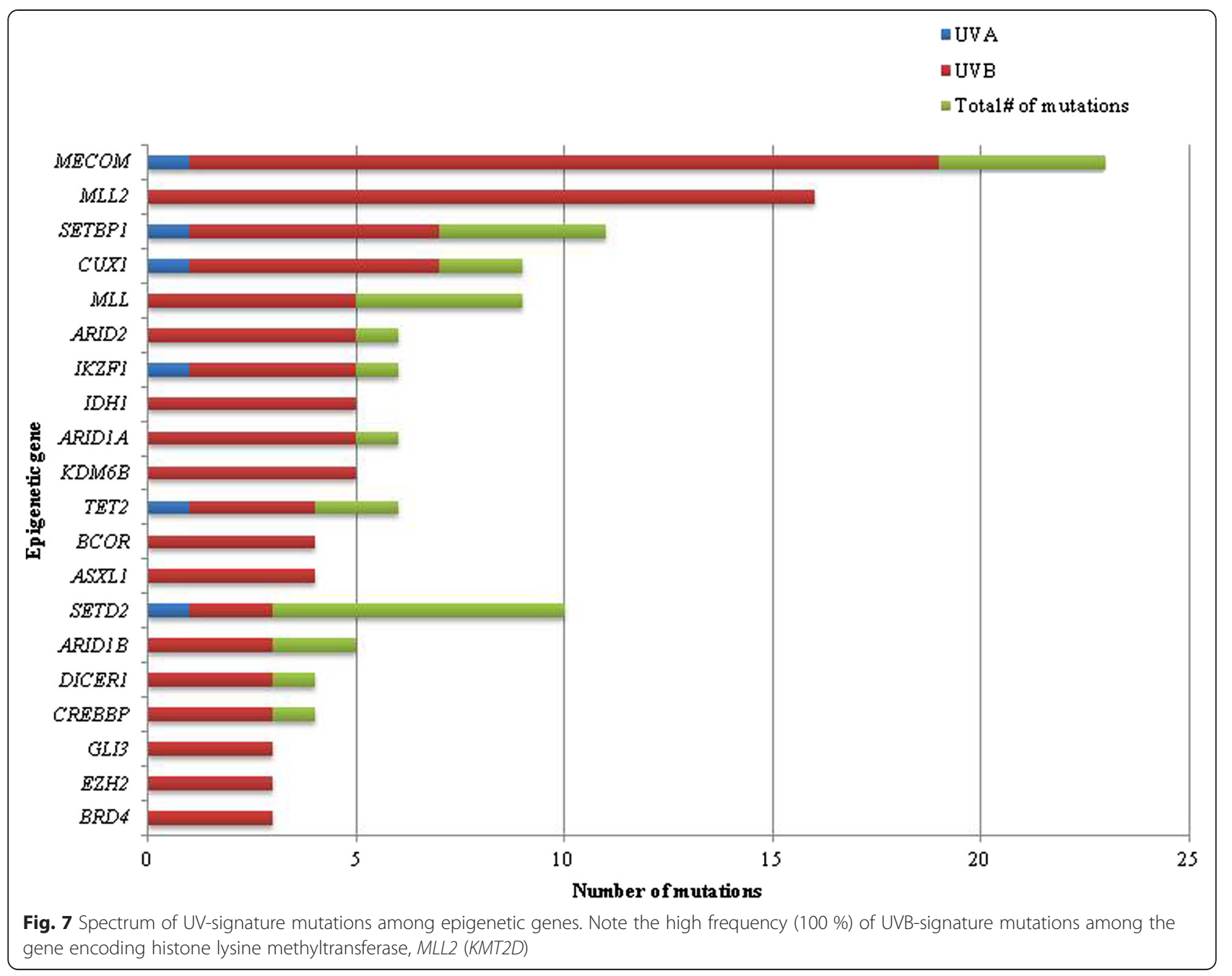

otherwise known as Fe (II)-dependent dioxygenase enzymes [44]. Furthermore, mutant IDH1 has been demonstrated to lead to the accumulation of oncometabolite 2-hydroxyglutarate (2-HG), which has been shown to directly inhibit TET function $[44,45]$. The TET family enzymes, composed of TET1, TET2, and TET3, are central to the active DNA demethylation pathway $[46,47]$. TET2 catalyzes the critical, iterative oxidation steps on the methyl group of 5-methylcytosine to produce 5-hydroxymethylcytosine, 5-carboxylcytosine, and 5-formylcytosine, and ultimately cytosine, after removal of the functional group by thymine DNA glycosylase and the base excision repair pathway [48]. This very recently uncovered pathway has putative significance for maintaining DNA methylation and epigenetic fidelity, which has earned TET the epithet "guardian of the epigenome" [12, 49].

5-hydroxymethylcytosine, an intermediate in DNA demethylation and a product of TET2 function is kinetically the most abundant intermediate in this pathway [50]. Loss of its nuclear immunopositivity has been demonstrated to be a nearly universal hallmark of malignancy [51]. A number of studies have demonstrated that, in most human organ systems, "loss of 5-hydroxymethylcytosine" can distinguish malignant cellular proliferations from the benign, wherein 5-hydroxymethylcytosine expression is retained, with high sensitivity and specificity [13, 51-53]. Such observations bear testament to the pathobiologic significance of TET enzyme dysfunction. Indeed, TET has recently been found to interact in concert with an array of epigenetic regulators, including transcription factors, histone modifying enzymes, chromatin-remodeling complexes, and miRNAs, suggesting a central regulatory role over the epigenome [54]. A recent study documented exceedingly rare somatic TET2 gene mutations in melanoma [55]. In contrast, six of 38 (15.8\%) of our human melanoma samples had a documented mutation in TET2. Moreover, when including IDH1-mutant samples, $21 \%$ (8 of 38) of patient samples had at least one mutation affecting the critical DNA demethylation pathway in which these two enzymes participate. That mutation to 


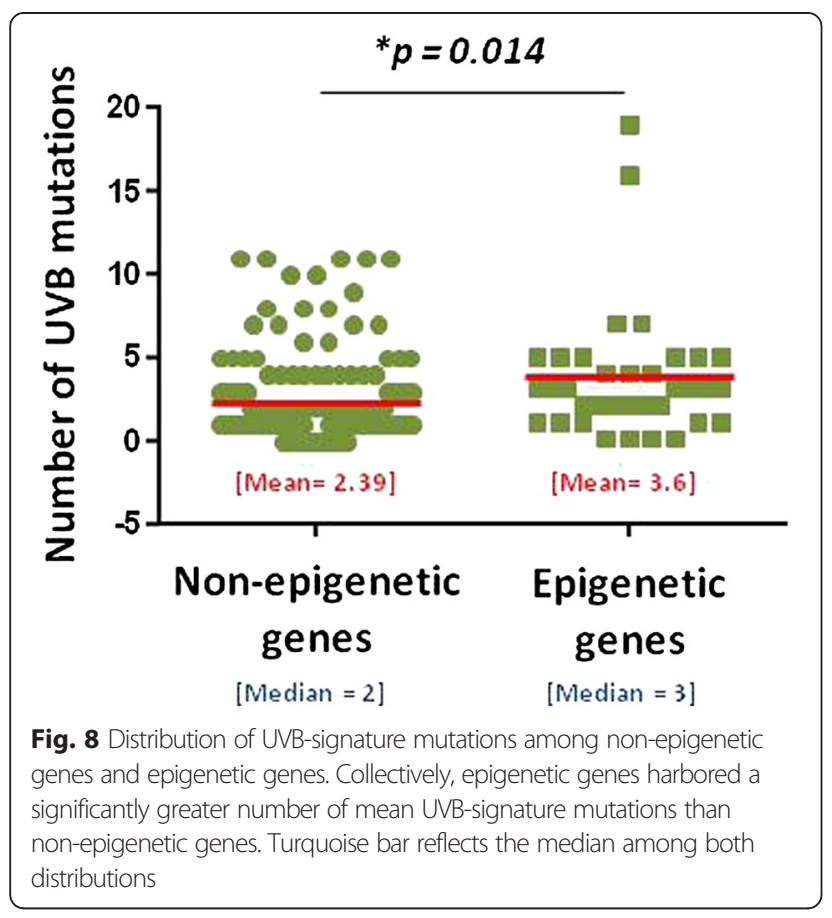

the gene encoding the TET2 enzyme is only one of many mechanisms that can cause its dysfunction [54] raises the possibility that additional alterations not detectable by genetic sequencing may, too, inhibit the function of this critical regulator of the epigenome and contribute to melanoma virulence.

Secondly, our dataset and analysis also identified a high frequency of UVB-signature mutations among epigenetic genes. The genes encoding the central epigenetic regulator MECOM/EVI1 (73.9 \%, 17 of 23) and H3K4 methyltranferase MLL2 (100\%, 16 of 16) were found to harbor the greatest overall number of UVB-signature mutations. Both within and outside of the familial/hereditary melanoma setting, ultraviolet light radiation (UVR) is thought to play a major role in the pathogenesis of melanoma. While it is known that most of the mutational burden in melanoma is attributable to the mutagenic effects of UVR [56, 57], our findings raise the possibility that UVR preferentially induces mutations in genes encoding epigenetic regulators and/or that these elements may be involved in mediating a physiologic

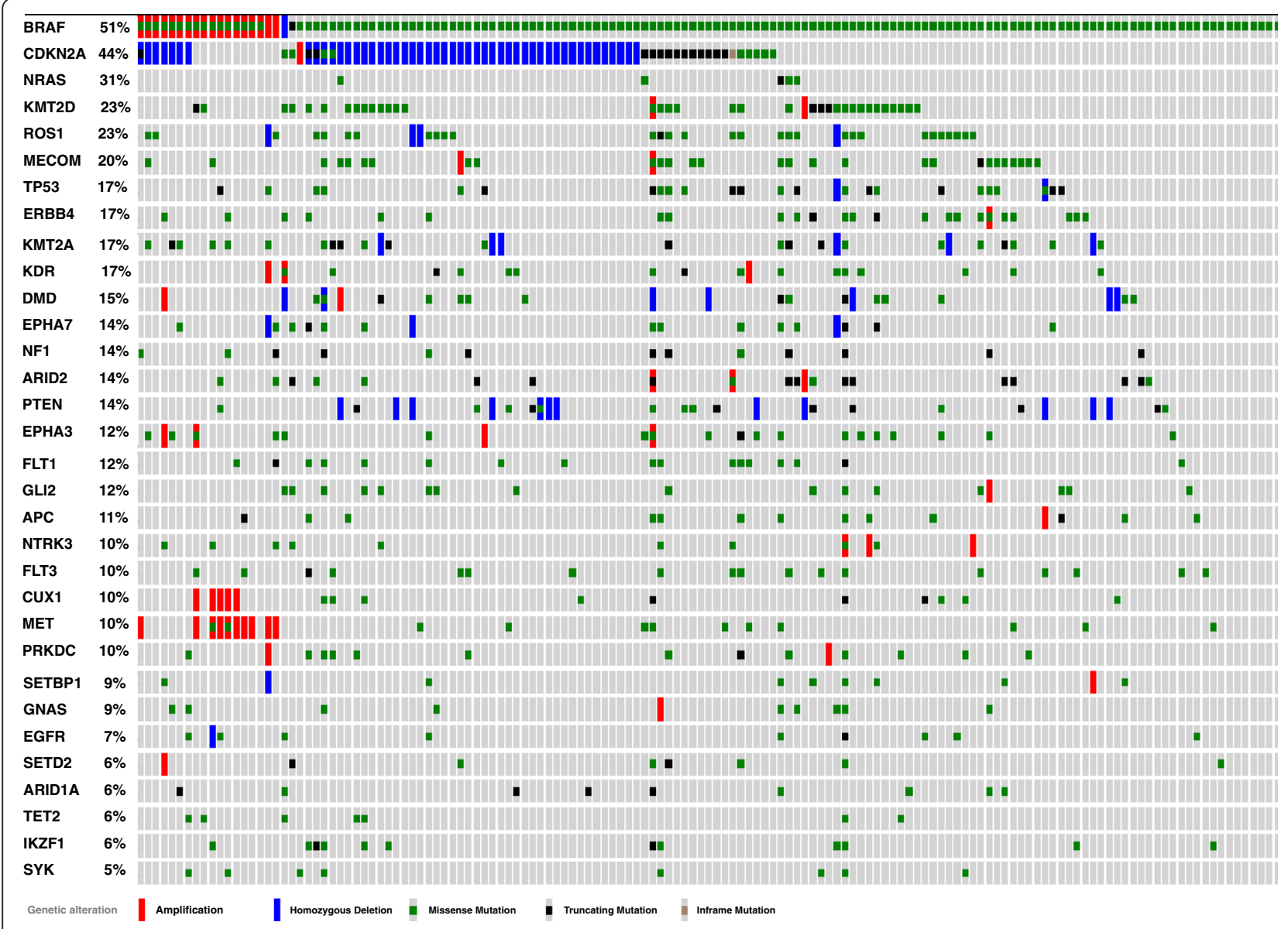

Fig. 9 Distribution of mutations in our "Top 40" genes among TCGA cutaneous melanomas ( $n=278)$ (data publicly accessible via http://www.cbioportal.org) 


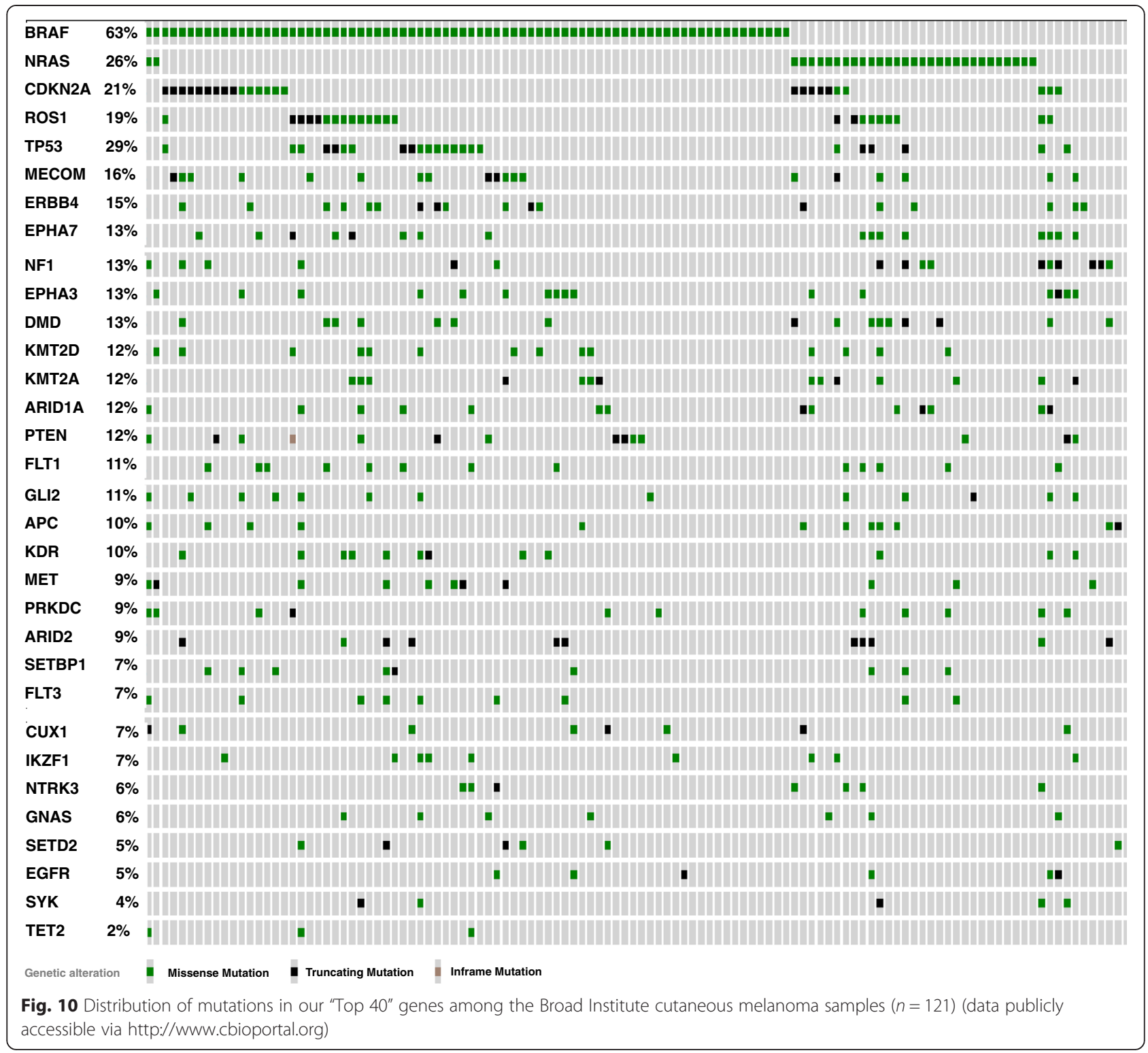

response. This hypothesis is in keeping with the putative role of epigenetic mechanisms at large in facilitating changes in gene expression in response to environmental cues $[12,58,59]$ and could co-exist with pathways known to be involved in the physiologic response of melanocytes to UVR, such as the p53-proopiomelanocortin (POMC) pathway [60]. Interestingly, we found that the well-known oncogenes BRAF and NRAS had comparatively low frequencies (26.3 and $0 \%$, respectively) of UVBsignature mutations, a finding that is in keeping with previous studies [20]. In contrast, $81.8 \%$ of $C D K N 2 A$ mutations, $84.6 \%$ of TP53 mutations, and $83.3 \%$ of IDH1 mutations resembled the UVB-signature mutation genotype in our cohort.

Genomic evidence that epigenetic regulators may contribute to melanoma pathogenesis was recently highlighted by Hodis et al. [20], who found a high frequency of somatic mutations in chromatin-modifying proteins and other epigenetic regulators as well as a high frequency of UVBsignature mutations in $I D H 1$ and chromatin-modifying enzymes ARID2 (a component of the SWI/SNF chromatinremodeling complex) and EZH2 (the histone lysine methylase component of Polycomb-group gene silencing complex). Ding et al. [31] also found a high frequency of truncation mutations to chromatin-remodeling genes (ASXL3, MLL2, ARID2) in their cohort of metastatic melanoma cases [31]. Our findings, in addition to data obtained from the COSMIC and TCGA melanoma database (Figs. 11 and 12), contribute additional evidence to this growing body of literature that dysregulated epigenetic complexes and pathways may be more involved in the pathobiology of melanoma than previously recognized. Our 


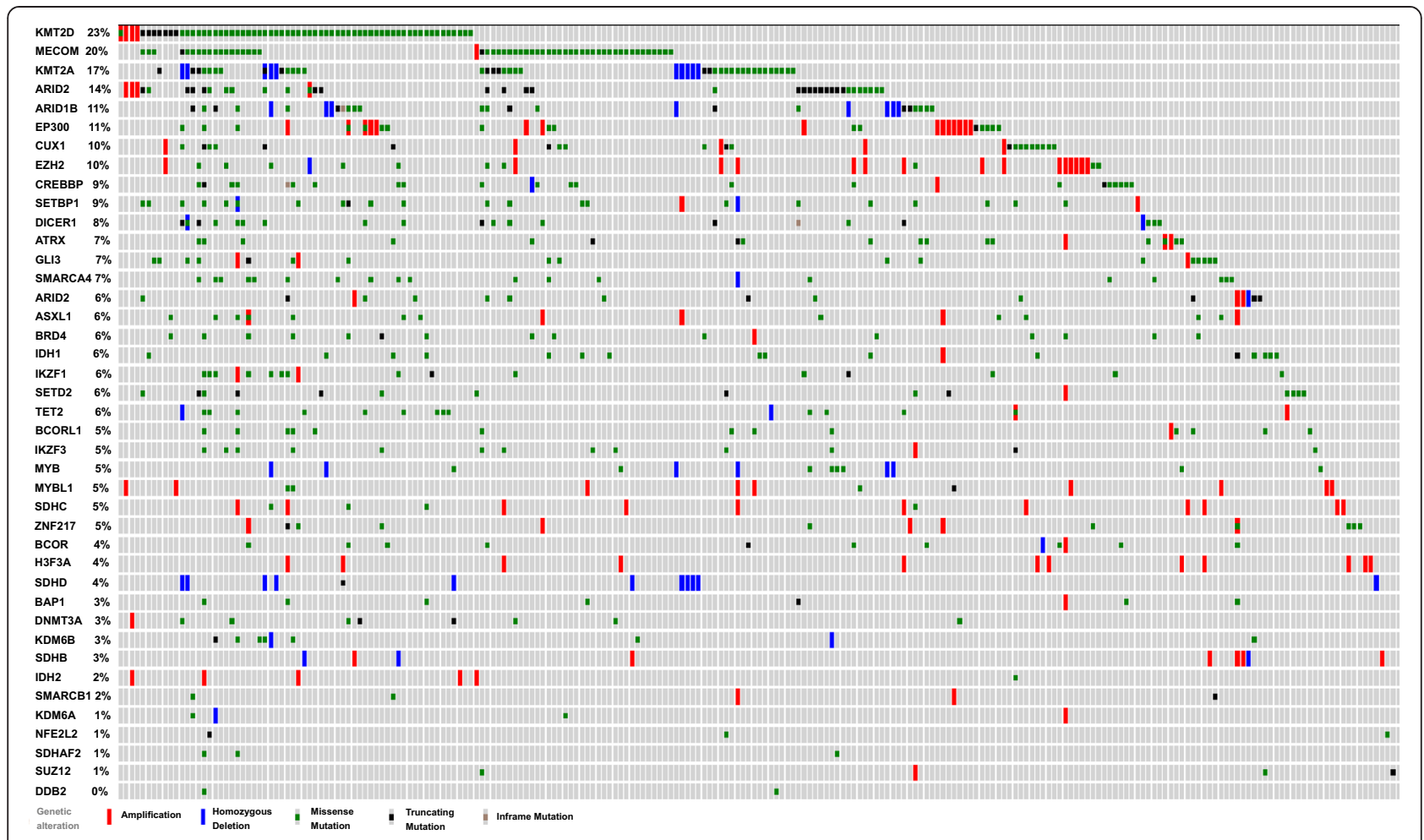

Fig. 11 Mutational spectrum among epigenetic genes tested in "Oncopanel" within TCGA cutaneous melanomas $(n=278)$ (data publicly accessible via http://www.cbioportal.org)

preliminary dataset indicate that genomic mutations to epigenetic regulators may be more common than previously appreciated. Altogether, we found that approximately one in five mutations occurred in a gene encoding an epigenetic regulator, with mutations to histone-modifying enzymes being the most common. Moreover, the overwhelming majority $(92.1 \%, 35$ of 38$)$ of our patient samples harbored at least one mutation in an epigenetic regulatory gene with well over half of all patients samples $(65.7 \%$, 25 of 38) having more than one such gene mutated.

While our study provides novel insight into potential epigenetic mechanisms involved in the pathogenesis of melanoma, there are several limitations. Firstly, our modestly sized cohort may under- or overestimate the frequency of certain genetic mutations and also limits our ability to identify a mutational signature, epigenetic or not, that is typical of primary or metastatic melanoma tumors or those that correlate with key histologic parameters that confer a defined level of metastatic potential (i.e., depth, mitotic rate). Further expansion and integration of inter-institutional patient melanoma mutational profiles alongside detailed, case-by-case clinical and histopathologic annotation will be necessary to obtain the resolution necessary to identify these critical relationships. Furthermore, the lack of paired samples within our cohort reduces our ability to identify or directly infer specific genetic mutations that potentially drive metastasis or disease progression. Given that the Oncopanel program at our institution is offered to every oncology patient but is programmatically limited to one test per patient due to economic constraints, our patient samples are unable to be paired. In addition, the panel of epigenetic genes tested in our cohort is not comprehensive, in view of the ever-expanding family of epigenomic regulators [61], and additional sequencing platforms to test for these novel epigenetic genes should be prepared for further investigations. It must also be acknowledged that sequencing techniques do not directly detect chromosomal aberrations, which are known to occur and be involved in the pathogenesis of melanoma [62] and distinguish benign melanocytic lesions from malignant melanoma [63]. Nonetheless, our study highlights the high prevalence of mutations in epigenetic regulators in patient melanoma samples and their tendency to bear the signature of UVB damage. Moreover, our focus on utilizing genetic information to understand dysregulated epigenetic mechanisms in melanoma provides a novel analytical lens that deserves further consideration. Finally, our study demonstrates the clinical application of next-generation sequencing to identify novel mutations in melanoma and may shed light on new, personalized pathogenic mechanisms and unveil potential targets of therapeutic interest [21]. Because epigenetic defects, unlike genetic mutations, are potentially reversible, 


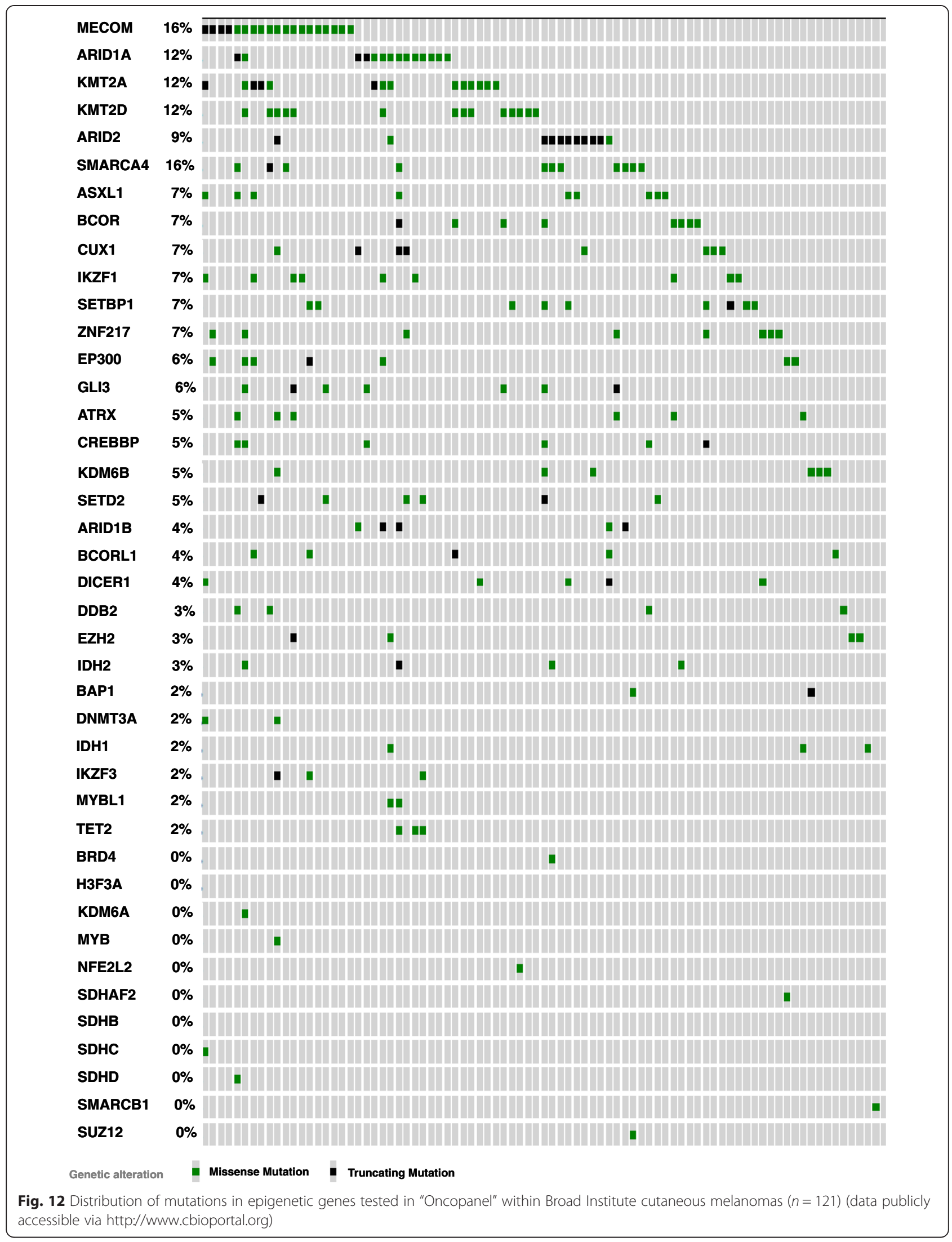


this area of investigation has tremendous potential for translational and therapeutic application.

Most existing non-immune based targeted therapies attempt to inhibit proteins/enzymes that function predominantly within and beneath the cell membrane and cytoplasm, most of which participate in cell signaling pathways $[64,65]$. Most such pathways are poised to influence the expression of select genes, a process that is primarily regulated by transcription factors and, very likely, other functional binding partners, such as chromatin-remodeling enzymes. As we continue to advance our understanding of how epigenetic mechanisms interface with cell signaling pathways and how their dysregulation, independently or combined, contributes to diseases such as cancer, opportunities for therapeutic targeting of pathobiologic epigenetic mechanisms will follow. In parallel, evidence is accumulating that multimodal combination therapy will be critical for the successful treatment of biologically complex malignancies such as melanoma [66]. Targeting epigenetic mechanisms may provide one such adjunctive avenue of attack, emphasizing the importance of delineating the relationships discussed above. Indeed, DNA methyltransferase inhibitors and histone deacetylase inhibitors are the only current examples of existing FDA-approved therapies that function in this manner, although they are not currently in use for melanoma. Our data highlight several candidate epigenetic regulators that deserve further investigation and pathobiological characterization in melanoma. Within our cohort, MECOM/EVI1, MLL2, and TET2/IDH are examples of nuclear epigenetic regulators that we suspect may be involved in the pathogenesis of melanoma and could be involved in enabling stem-like characteristics in select subpopulations. This epigenetic machinery deserves thorough exploration in the context of melanoma pathobiology, and further studies to establish their mutational status in benign nevi are now indicated.

\section{Conclusions}

Herein, we describe the prevalence of somatic mutations present in the genes encoding a spectrum of epigenetic regulators within a cohort of treatment-naïve patient melanoma specimens. We provide direct genomic evidence that epigenetic regulators, including histone/chromatin-modifying enzymes and DNA demethylation enzymes/ pathways may be involved in the development and/or progression of melanoma. Moreover, our analysis of patient melanoma samples revealed a high prevalence of mutations in epigenetic regulators with a quantifiable predilection for those associated with UVB damage. In addition, we have identified that $M E C O M$, a novel, central epigenetic regulatory gene, and TET2/IDH1, critical regulators of DNA demethylation, are frequently mutated in patient melanoma samples.

\section{Availability of supporting data}

The datasets supporting the results of this article are included within the article and its additional file.

\section{Methods}

\section{Ethics statement}

The study was approved by the Institutional Review Board of the Brigham and Women's Hospital and Dana-Farber Cancer Institute. Patients referred to our institution for the evaluation and management of melanoma were offered an opportunity to participate in this study. Informed consent was obtained from 38 patients to collect a single sample of their primary or metastatic tumor and have it sequenced through the Oncopanel program. Oncopanel was designed and implemented to provide an opportunity for every oncology patient seen and evaluated at the institution to have their tumor tested. However, because its use is not currently being reimbursed, it is programmatically limited to only one test per patient at the present time. All 38 patients had not previously received any form of chemotherapy or radiation for the treatment of their melanoma.

DNA was isolated from formalin-fixed melanoma tissue using standard methods. Samples were incubated in proteinase $\mathrm{K}$ overnight, followed by subsequent purification of the DNA (QIAamp DNA Mini Kit, QIAGEN, Gaithersburg, MD). DNA concentration was assessed using PicoGreen dsDNA detection (Life Technologies, Carlsbad, CA). Targeted NGS was performed using a cancer genomic assay to detect mutations in the exonic regions of 275 cancer genes previously implicated in tumorigenesis and 91 intronic regions across 30 of the 275 genes (Oncopanel, BWH/DFCI, Additional file 1). The complete coding sequence of the target genes was captured using a solution-phase Agilent SureSelect hybrid capture kit (AgilentTechnologies, Inc., Santa Clara, $\mathrm{CA}$ ), and massively parallel sequencing was performed on an Illumina HiSeq 2500 sequencer (Illumina, Inc. San Diego, CA). Mutation calls were performed using Mutect and GATK software (Broad Institute, Cambridge, MA).

Data analysis was performed using an internally developed bioinformatics Pipeline (Riker, REF) that was composed of reconfigured publically available tools (GATK, MuTect, Indelocator, Oncotator) and internally developed algorithms (VisCap Cancer [REF], Phaser, BreaKmer3). Reads obtained from pooled samples were demultiplexed using Picard (http://picard.sourceforge.net/command-lineoverview.html), aligned to the Human Genome Reference Consortium reference sequence GRCh37p13 (BWA5), and duplicate reads were subsequently removed (Picard). GATK6 was used to refine the alignments near insertion/ deletion (indel) sites. Single nucleotide variants (SNVs) were called using MuTect7, and indels were called using Indelocator (http://www.broadinstitute.org/cancer/ 
cga/indelocator). Annotation was performed using Oncotator. Because tumor tissues were sequenced without a paired normal from the corresponding patients, additional informatic steps were taken to identify and account for common single nucleotide polymorphisms (SNPS): any SNP present at $>0.1 \%$ in Exome Variant Server (NHLBI GO Exome Sequencing Project [ESP], Seattle, WA; URL: http://evs.gs.washington.edu/EVS/) or present in dbSNP was filtered. However, variants also present in the COSMIC mutation database were rescued for manual review. Samples with a mean target coverage of $<50 \mathrm{X}$ were failed and excluded from further analysis. Individual variants present at $<10 \%$ allele fraction or in regions with $<50 \mathrm{X}$ coverage were flagged for manual review and evaluated/interpreted by the reviewing laboratory scientists and molecular pathologists based on a variety of factors, including, but not limited to, overall tumor percentage, read depth, complexity of alteration, and evidence for associated copy number alterations.

Mutation data was queried with Microsoft Sequel and quantitatively analyzed with Microsoft Excel. Individual gene mutation frequencies were calculated based on the total number of mutations as well as based on the total number of patients. Genes encoding proteins involved in DNA methylation/demethylation, histone modification, chromatin remodeling, or processing of non-coding RNAs (such as microRNAs) were categorized as "epigenetic regulatory genes". Gene functions were determined by referencing the genetic database (accessible at: http:// ghr.nlm.nih.gov/) provided by the United States National Library of Medicine and the National Institute of Health and supplemented by identifying recently published literature available on PubMed documenting specific epigenetic function by a particular gene or its expressed protein. Quantitative data was analyzed using MedCalc version 13.2 (MedCalc Software, Ostend, Belgium) and StatPlus version 5.8.2 (AnalystSoft Inc.). Statistical methods were primarily descriptive and based on proportions and percentages. The proportion of epigenetic genes is presented with a $95 \%$ exact binomial confidence interval. Proportions of epigenetic genes or mutations present within particular cohorts were compared using chi-square analysis. All $p$ values were two-sided, with a $p<0.05$ considered statistically significant.

Additional mutation data was obtained from the publically available databases (http://www.cbioportal.org, Memorial Sloan Kettering Cancer Center, New York, NY) provided by the National Institute of Health $(\mathrm{NIH})$ and National Cancer Institute (NCI) for the purposes of comparison [22, 23]. In addition, to better understand the relationships between mutated epigenetic regulators, we performed Ingenuity Pathway Analysis (Qiagen, Redwood City, CA) to visualize direct relationships between specific epigenetic regulators.

\section{Additional file}

Additional file 1: List of the $\mathbf{2 7 5}$ cancer gene exons as well as the 91 introns from 30 of these genes sequenced by our "Oncopanel" cancer genomic assay.

\section{Abbreviations}

a-KG: alpha-ketoglutarate; 5-mC: 5-methylcytosine; 5-hmC:

5-hydroxymethylcytosine; 5-caC: 5-carboxylcytosine; ARID1B: AT rich interactive domain 1B (SWI1-like); ARID2: AT rich interactive domain 2 (RFX-like); ASB2: ankyrin repeat and SOCS box protein 2; ASXL3: additional sex combs like 3; BAP1: BRCA1 associated protein-1 (ubiquitin carboxy-terminal hydrolase); BRAF: B-Raf proto-oncogene, serine/threonine kinase; BWH: Brigham and Women's Hospital; C: cytosine; CDK4: cyclin-dependent kinase 4; CDKN2A: cyclin-dependent kinase inhibitor 2A; CREBBP: CREB binding protein; COSMIC: Catalogue of Somatic Mutations in Cancer; CUX1: cut-like homeobox 1; DFCl: Dana-Faber Cancer Institute DNA, Deoxyribonucleic acid; DNMT: DNA methyltransferase; EZH2: enhancer of zeste homolog 2; FDA: United States Food and Drug Administration; G: guanine; HDAC: histone deacetylase; IDH: gene encoding isocitrate dehydrogenase; KMTD: lysine (K)-specific methyltransferase 2D; KMT2A: lysine (K)-specific methyltransferase 2A; MECOM: MDS1 and EV1 complex locus; MLL(2): mixed-lineage leukemia protein (2) miRNA, MicroRNA; NCl: National Cancer Institute; NF1: neurofibromin 1; NGS: next-generation sequencing; $\mathrm{NIH}$ : National Institute of Health; NRAS: neuroblastoma RAS viral (v-ras) oncogene homolog; POMC: p53-proopiomelanocortin; POT1: protection of telomeres 1; SETD2: SET domain containing 2; SWI/SNF: SWItch/Sucrose NonFermentable; T: thymine; TCGA: The Cancer Genome Atlas;

TERT: telomerase reverse transcriptase; TET: ten-eleven translocase;

TP53: tumor protein p53; UVR: ultraviolet radiation.

\section{Competing interests}

The authors declare that they have no competing interests.

\section{Authors' contributions}

$J \mathrm{~J}, \mathrm{LMS}, \mathrm{GFM}$, and CGL designed the study and performed the primary analysis of the data. NIL, PS, and GC oversaw the sequencing of the samples, analysis, and writing of the manuscript. JJL, PAO, NB, FSH, MCM, AEW, HAH, $J Y L, S R G$, and $A C L$, oversaw the clinical program for melanoma patients at the Dana-Farber Cancer Institute, assisted in obtaining and interpreting the samples for analysis, and provided critical feedback on the analysis and writing of the manuscript. RL provided technical assistance in the analysis of data as well as the preparation of figures and the manuscript. All authors read and approved the final manuscript.

\section{Acknowledgements}

This study was supported by NIH grant 5P40CA093683-09 to the SPORE Core at Brigham and Women's Hospital. GFM received funding from Bristol-Myers-Squibb for work on melanoma biomarkers (not a funding source for this study). This work is also supported by Harvard Medical School Eleanor and Miles Shore Fellowship Program (CGL) and Karin Grunebaum Cancer Research Foundation (CGL). This work was conducted with support from Harvard Catalyst/The Harvard Clinical and Translational Science Center (NCRR and NCATS, NIH Award UL1 TR001102) and financial contributions from Harvard University and its affiliated academic healthcare centers.

\section{Author details}

'Department of Pathology, Brigham and Women's Hospital, Harvard Medical School, 221 Longwood Avenue, EBRC Suite 401, Boston, MA 02115, USA. ${ }^{2}$ Melanoma Center, Dana-Farber Cancer Institute, Harvard Medical School, 450 Brookline Ave., Boston, MA 02215-5450, USA.

Received: 17 February 2015 Accepted: 27 May 2015

Published online: 09 June 2015

\section{References}

1. Lian CG, Mihm MC, Pierard G, Tommasino M. Skin Cancer. In: Stewart BW, Wild CP, editors. World Cancer Report 2014. Lyon, France: International Agency for Research on Cancer; 2014. 
2. Davies H, Bignell GR, Cox C, Stephens P, Edkins S, Clegg S, et al. Mutations of the BRAF gene in human cancer. Nature. 2002;417:949-54.

3. Chapman PB, Hauschild A, Robert C, Haanen JB, Ascierto P, Larkin J, et al. Improved survival with vemurafenib in melanoma with BRAF V600E mutation. N Engl J Med. 2011;364:2507-16.

4. Pollock PM, Harper UL, Hansen KS, Yudt LM, Stark M, Robbins CM, et al. High frequency of BRAF mutations in nevi. Nat Genet. 2003;33:19-20.

5. Garraway LA, Chin L. Chapter 118: molecular biology of cutaneous melanoma In: Devita J VT, Lawrence TS, Rosenberg SA, editors. Cancer: Principles \& Practice of Oncology, 9e. Philadelphia, PA: Lippincott Williams \& Wilkins; 2011.

6. Swanton C, Andre F, Mardis E. Deciphering root causes of intrinsic BRAF inhibitor resistance in melanoma: ushering in a new genomics case reports feature for Annals of Oncology. Ann Oncol. 2014;25:917-8.

7. Chapman PB, Solit DB, Rosen N. Combination of RAF and MEK inhibition for the treatment of BRAF-mutated melanoma: feedback is not encouraged. Cancer Cell. 2014;26:603-4.

8. Flaherty KT, Infante JR, Daud A, Gonzalez R, Kefford RF, Sosman J, et al. Combined BRAF and MEK inhibition in melanoma with BRAF V600 mutations. N Engl J Med. 2012;367:1694-703.

9. Flaherty KT, Robert C, Hersey P, Nathan P, Garbe C, Milhem M, et al. Improved survival with MEK inhibition in BRAF-mutated melanoma. N Engl J Med. 2012;367:107-14.

10. Hodi FS, O'day SJ, Mcdermott DF, Weber RW, Sosman JA, Haanen JB, et al. Improved survival with ipilimumab in patients with metastatic melanoma. N Engl J Med. 2010;363:711-23.

11. Robert C, Long GV, Brady B, Dutriaux C, Maio M, Mortier L, et al. Nivolumab in previously untreated melanoma without BRAF mutation. N Engl J Med. 2015;372:320-30

12. Lee JJ, Murphy GF, Lian CG. Melanoma epigenetics: novel mechanisms, markers, and medicines. Lab Invest. 2014;94:822-38.

13. Lian CG, Xu Y, Ceol C, Wu F, Larson A, Dresser K, et al. Loss of 5-hydroxymethylcytosine is an epigenetic hallmark of melanoma. Cell. 2012;150:1135-46.

14. Zingg D, Debbache J, Schaefer SM, Tuncer E, Frommel SC, Cheng P, et al. The epigenetic modifier EZH2 controls melanoma growth and metastasis through silencing of distinct tumour suppressors. Nat Commun. 2015;6:6051.

15. De Raedt T, Beert E, Pasmant E, Luscan A, Brems H, Ortonne N, et al. PRC2 loss amplifies Ras-driven transcription and confers sensitivity to BRD4-based therapies. Nature. 2014;514:247-51.

16. Sullivan RJ, Flaherty K. MAP kinase signaling and inhibition in melanoma. Oncogene. 2013;32:2373-9.

17. Turajlic S, Furney SJ, Stamp G, Rana S, Ricken G, Oduko Y, et al. Whole-genome sequencing reveals complex mechanisms of intrinsic resistance to BRAF inhibition. Ann Oncol. 2014;25:959-67.

18. Kaufman HL, Kirkwood JM, Hodi FS, Agarwala S, Amatruda T, Bines SD, et al. The Society for Immunotherapy of Cancer consensus statement on tumour immunotherapy for the treatment of cutaneous melanoma. Nat Rev Clin Oncol. 2013;10:588-98.

19. Van Allen EM, Wagle N, Sucker A, Treacy DJ, Johannessen CM, Goetz EM, et al. The genetic landscape of clinical resistance to RAF inhibition in metastatic melanoma. Cancer Discov. 2014:4:94-109.

20. Hodis E, Watson IR, Kryukov GV, Arold ST, Imielinski M, Theurillat JP, et al. A landscape of driver mutations in melanoma. Cell. 2012;150:251-63.

21. Griewank KG, Scolyer RA, Thompson JF, Flaherty KT, Schadendorf D, Murali R. Genetic alterations and personalized medicine in melanoma: progress and future prospects. J Natl Cancer Inst. 2014;106:djt435.

22. Gao J, Aksoy BA, Dogrusoz U, Dresdner G, Gross B, Sumer SO, et al. Integrative analysis of complex cancer genomics and clinical profiles using the cBioPortal. Sci Signal. 2013;6:pl1.

23. Cerami E, Gao J, Dogrusoz U, Gross BE, Sumer SO, Aksoy BA, et al. The cBio cancer genomics portal: an open platform for exploring multidimensional cancer genomics data. Cancer Discov. 2012;2:401-4.

24. Vogel Rl, Ahmed RL, Nelson HH, Berwick M, Weinstock MA, Lazovich D. Exposure to indoor tanning without burning and melanoma risk by sunburn history. J Natl Cancer Inst. 2014;106

25. Sanlorenzo M, Wehner MR, Linos E, Kornak J, Kainz W, Posch C, et al. The risk of melanoma in airline pilots and cabin crew: a meta-analysis. JAMA Dermatol. 2015;151:51-8.

26. Huang FW, Hodis E, Xu MJ, Kryukov GV, Chin L, Garraway LA. Highly recurrent TERT promoter mutations in human melanoma. Science. 2013;339:957-9.
27. Shi J, Yang XR, Ballew B, Rotunno M, Calista D, Fargnoli MC, et al. Rare missense variants in POT1 predispose to familial cutaneous malignant melanoma. Nat Genet. 2014;46:482-6.

28. Tucker MA, Fraser MC, Goldstein AM, Struewing JP, King MA, Crawford JT, et al. A natural history of melanomas and dysplastic nevi: an atlas of lesions in melanoma-prone families. Cancer. 2002;94:3192-209.

29. Jeck WR, Parker J, Carson CC, Shields JM, Sambade MJ, Peters EC, et al. Targeted next generation sequencing identifies clinically actionable mutations in patients with melanoma. Pigment Cell Melanoma Res. 2014;27:653-63.

30. Bamford S, Dawson E, Forbes S, Clements J, Pettett R, Dogan A, et al. The COSMIC (Catalogue of Somatic Mutations in Cancer) database and website. Br J Cancer. 2004:91:355-8.

31. Ding L, Kim M, Kanchi KL, Dees ND, Lu C, Griffith M, et al. Clonal architectures and driver mutations in metastatic melanomas. PLoS One. 2014;9:e111153.

32. Chin L, Garraway LA, Fisher DE. Malignant melanoma: genetics and therapeutics in the genomic era. Genes Dev. 2006;20:2149-82.

33. Lugthart S, Van Drunen E, Van Norden Y, Van Hoven A, Erpelinck CA, Valk PJ, et al. High EVI1 levels predict adverse outcome in acute myeloid leukemia: prevalence of EVI1 overexpression and chromosome $3 \mathrm{q} 26$ abnormalities underestimated. Blood. 2008;111:4329-37.

34. Groschel S, Schlenk RF, Engelmann J, Rockova V, Teleanu V, Kuhn MW, et al. Deregulated expression of EVI1 defines a poor prognostic subset of MLL-rearranged acute myeloid leukemias: a study of the German-Austrian Acute Myeloid Leukemia Study Group and the Dutch-Belgian-Swiss HOVON/SAKK Cooperative Group. J Clin Oncol. 2013;31:95-103.

35. Bard-chapeau EA, Gunaratne J, Kumar P, Chua BQ, Muller J, Bard FA, et al. EVI1 oncoprotein interacts with a large and complex network of proteins and integrates signals through protein phosphorylation. Proc Natl Acad Sci U S A. 2013;110:E2885-94.

36. Kataoka K, Kurokawa M. Ecotropic viral integration site 1, stem cell self-renewal and leukemogenesis. Cancer Sci. 2012;103:1371-7.

37. Rommer A, Steinmetz B, Herbst F, Hackl H, Heffeter P, Heilos D, et al. EVI1 inhibits apoptosis induced by antileukemic drugs via upregulation of CDKN1A/p21/WAF in human myeloid cells. PLoS One. 2013;8:e56308.

38. Ruthenburg AJ, Allis CD, Wysocka J. Methylation of lysine 4 on histone H3: intricacy of writing and reading a single epigenetic mark. Mol Cell. 2007;25:15-30.

39. Guo C, Chang CC, Wortham M, Chen LH, Kernagis DN, Qin X, et al. Global identification of MLL2-targeted loci reveals MLL2's role in diverse signaling pathways. Proc Natl Acad Sci U S A. 2012;109:17603-8.

40. Gao YB, Chen ZL, Li JG, Hu XD, Shi XJ, Sun ZM, et al. Genetic landscape of esophageal squamous cell carcinoma. Nat Genet. 2014;46:1097-102.

41. Yin $S$, Yang J, Lin B, Deng $W$, Zhang $Y$, Yi $X$, et al. Exome sequencing identifies frequent mutation of MLL2 in non-small cell lung carcinoma from Chinese patients. Sci Rep. 2014;4:6036.

42. Ross JS, Wang K, Elkadi OR, Tarasen A, Foulke L, Sheehan CE, et al. Next-generation sequencing reveals frequent consistent genomic alterations in small cell undifferentiated lung cancer. J Clin Pathol. 2014;67:772-6.

43. Yan H, Parsons DW, Jin G, Mclendon R, Rasheed BA, Yuan W, et al. IDH1 and IDH2 mutations in gliomas. N Engl J Med. 2009;360:765-73.

44. Losman JA, Kaelin Jr WG. What a difference a hydroxyl makes: mutant IDH, (R)-2-hydroxyglutarate, and cancer. Genes Dev. 2013;27:836-52.

45. Reitman ZJ, Yan H. Isocitrate dehydrogenase 1 and 2 mutations in cancer: alterations at a crossroads of cellular metabolism. J Natl Cancer Inst. 2010;102:932-41.

46. He YF, Li BZ, Li Z, Liu P, Wang Y, Tang Q, et al. Tet-mediated formation of 5-carboxylcytosine and its excision by TDG in mammalian DNA. Science. 2011:333:1303-7.

47. Ito S, Shen L, Dai Q, Wu SC, Collins LB, Swenberg JA, et al. Tet proteins can convert 5-methylcytosine to 5-formylcytosine and 5-carboxylcytosine. Science. 2011;333:1300-3.

48. Nabel CS, Kohli RM. Molecular biology. Demystifying DNA demethylation. Science. 2011;333:1229-30.

49. Williams K, Christensen J, Helin K. DNA methylation: TET proteins - guardians of CpG islands? EMBOR Rep. 2012;13:28-35.

50. Salvaing J, Aguirre-Lavin T, Boulesteix C, Lehmann G, Debey P, Beaujean N. 5-Methylcytosine and 5-hydroxymethylcytosine spatiotemporal profiles in the mouse zygote. PLoS One. 2012;7:e38156.

51. Haffner MC, Chaux A, Meeker AK, Esopi DM, Gerber J, Pellakuru LG, et al. Global 5-hydroxymethylcytosine content is significantly reduced in tissue stem/progenitor cell compartments and in human cancers. Oncotarget. 2011;2:627-37. 
52. Uchiyama R, Uhara H, Uchiyama A, Ogawa E, Takazawa Y, Ashida A, et al. 5-Hydroxymethylcytosine as a useful marker to differentiate between malignant melanomas and benign melanocytic nevi. J Dermatol Sci. 2014;73:161-3.

53. Lee JJ, Granter SR, Laga AC, Saavedra AP, Zhan Q, Guo W, et al. 5-Hydroxymethylcytosine expression in metastatic melanoma versus nodal nevus in sentinel lymph node biopsies. Mod Pathol. 2015;28:218-29.

54. Roy DM, Walsh LA, Chan TA. Driver mutations of cancer epigenomes. Protein Cell. 2014;5:265-96.

55. Song $\mathrm{F}$, Amos $\mathrm{Cl}$, Lee JE, Lian CG, Fang S, Liu H, et al. Identification of a melanoma susceptibility locus and somatic mutation in TET2. Carcinogenesis. 2014;35:2097-101.

56. Liu JJ, Fisher DE. Lighting a path to pigmentation: mechanisms of MITF induction by UV. Pigment Cell Melanoma Res. 2010;23:741-5.

57. Tran TT, Schulman J, Fisher DE. UV and pigmentation: molecular mechanisms and social controversies. Pigment Cell Melanoma Res. 2008;21:509-16.

58. Somers EC, Richardson BC. Environmental exposures, epigenetic changes and the risk of lupus. Lupus. 2014;23:568-76.

59. Questa Jl, Walbot V, Casati P. Mutator transposon activation after UV-B involves chromatin remodeling. Epigenetics. 2010;5:352-63.

60. Lo JA, Fisher DE. The melanoma revolution: from UV carcinogenesis to a new era in therapeutics. Science. 2014;346:945-9.

61. Huether R, Dong L, Chen X, Wu G, Parker M, Wei L, et al. The landscape of somatic mutations in epigenetic regulators across 1,000 paediatric cancer genomes. Nat Commun. 2014;5:3630.

62. Kwong LN, Chin L. Chromosome 10, frequently lost in human melanoma, encodes multiple tumor-suppressive functions. Cancer Res. 2014;74:1814-21.

63. Bastian BC. Understanding the progression of melanocytic neoplasia using genomic analysis: from fields to cancer. Oncogene. 2003;22:3081-6.

64. Sullivan RJ, Fisher DE. Understanding the biology of melanoma and therapeutic implications. Hematol Oncol Clin North Am. 2014;28:437-53.

65. Smalley KS, Weber JS. Up close and personal: the challenges of precision medicine in melanoma. J Natl Cancer Inst. 2014;106:djt443.

66. Bozic I, Reiter JG, Allen B, Antal T, Chatterjee K, Shah P, et al. Evolutionary dynamics of cancer in response to targeted combination therapy. Elife. 2013;2:e00747.

\section{Submit your next manuscript to BioMed Central and take full advantage of:}

- Convenient online submission

- Thorough peer review

- No space constraints or color figure charges

- Immediate publication on acceptance

- Inclusion in PubMed, CAS, Scopus and Google Scholar

- Research which is freely available for redistribution 